\title{
Purification of Curcuminoids from Natural Deep Eutectic Solvents (NADES) Matrices Using Chromatography-Based Separation Methods
}

\author{
Orchidea Rachmaniah*, Muhammad Rifqy Muhsin, Angga Widya Putra, and Muhammad \\ Rachimoellah
}

Department of Chemical Engineering, Faculty of Industrial Technology and System Engineering, Institut Teknologi Sepuluh Nopember, Kampus ITS Sukolilo, Surabaya 60111, Indonesia

\section{* Corresponding author:}

tel: $+62-81233777793$

email:orchidea@chem-eng.its.ac.id

Received: August 21, 2020

Accepted: April 7, 2021

DOI: $10.22146 / \mathrm{ijc} .58935$

\begin{abstract}
Curcuminoids can be successfully extracted from Curcuma zedoaria using natural deep eutectic solvents (NADES) as extraction solvents. However, a mixture of extracted curcuminoids, NADES, and impurities from C. zedoaria was obtained as a slurry at the end of the extraction process. Therefore, further separation and purification were required to obtain the extracted compound in high purity. Herein, two purification methods based on classical column chromatography (CCC) and solid-phase extraction (SPE), were evaluated for the purification of curcuminoids from NADES matrices after extraction. Choline chloride-malic acid-water $\left(\mathrm{CCMA}-\mathrm{H}_{2} \mathrm{O}\right)$ and choline chloride-citric acid-water $\left(\mathrm{CCCA}-\mathrm{H}_{2} \mathrm{O}\right)$ in the molar ratio of 1:1:18 were selected as NADES matrices due to their high solubility and stabilization capability for curcuminoids. Ethanolconditioned silica gel (60-200 $\mu \mathrm{m})$ was applied as the bed resin for CCC, and a $C_{18}$ cartridge was used for SPE. Acetonitrile $/ 0.1 \%$ acetic acid, water $/ 0.1 \%$ acetic acid, and isopropanol $/ 0.1 \%$ acetic acid were used as mobile phases for CCC. For SPE, methanol/0.05\% acetic acid and water $0.05 \%$ acetic acid were applied in the conditioning step, water $/ 0.05 \%$ acetic acid in the washing step, and methanol/acetonitrile (1:1) in the eluting step. The SPE method produced higher recovery of curcuminoids from the CCCA$\mathrm{H}_{2} \mathrm{O}$ and CCMA- $\mathrm{H}_{2} \mathrm{O}$ matrices (75.27\% and $73.40 \%$, respectively) compared to CCC (51.9\% and $61.0 \%$, respectively). After removing the NADES constituents from the crude extract of curcuminoids, recrystallization was attempted.
\end{abstract}

Keywords: curcumin; chromatography; recrystallization; separation; solid phase extraction

\section{- INTRODUCTION}

Natural deep eutectic solvents (NADES) have attracted a great deal of attention since their first introduction in the third quarter of 2011 [1]. The NADES consist of a solution mixture of primary metabolites of hydrogen-bond acceptor (HBA) and hydrogen-bond donor (HBD) compounds such as sugars, organic acids, amino acids, and polyalcohol, which, in certain proportions, can act as a solvent for solubilizing, storing, or even transporting non-water-soluble compounds in organisms [1].

The concept of NADES is similar to that of ionic liquids (ILs) and deep eutectic solvents (DES). Ionic liquids are made of high purity synthetic organic chemicals containing organic cations and either organic or inorganic anions. Meanwhile, DES are mixtures of salts and/or organic compounds that, in certain proportions, can result in the formation of a liquid with entirely new properties that differ completely from those of the pure constituents. The DES can be readily prepared by mixing an HBA and an HBD compounds such as choline chloride and an organic acid, respectively, in eutectic proportion. For instance, mixtures of choline chloride and organic acids such as oxalic, malonic, and succinic acids were introduced in 2001 by Abbot [2]. The same group also reported other combinations of chemical compounds for DES in 2003 [2]. Another DES was prepared by combining choline chloride with alcohol

Orchidea Rachmaniah et al. 
[3]. As environmentally friendly solvents, NADES offer various advantageous over DES, especially for food, cosmetic, and pharmaceutical applications.

The solubilizing capacity of various NADES including proline/malic acid $(\mathrm{PMH})$, sucrose-choline chloride, glucose-choline chloride, sorbitol-choline chloride, 1,2-propanediol-choline chloride, fructoseglucose-sucrose, and glucose-acid lactate (LGH) for a wide range of compounds was explored by Dai [4]. The non-water-soluble compounds such as gluten, starch, DNA, cinnamic acid, carthamin, quercetin, and rutin proved to be soluble in NADES as well as in macromolecules including proteins and polysaccharides. Their solubility in NADES was substantially higher than that in water. Apart from carthamin, NADES also showed high solubilizing power for other phenolic metabolites in Carthamus tinctorius L., i.e., cartormin [5]. Cartormin and carthamin exhibited the highest solubility in $\mathrm{PMH}$ and LGH, and both metabolites were highly stable in sugar-based NADES when exposed to light, high temperatures, and long storage times [3-4]. The extraction of other phenolic compounds using NADES as a solvent was also reported, e.g., curcuminoids from Curcuma longa [6] and from C. zedoaria [7-8], polyphenolics from food by-products such as olive [9] and grape pomace [10], hydroxycinnamic acid and flavonoids from Moringa oleifera [11], phenylpropanoids and flavonoids from Lippia citriodora [12], and other polyphenolic compounds from peppermint (Mentha piperita) leaves and dried lemon balm (Melissa officinalis) leaves [13]. The nature and chemical structure of the phenolic compounds were found to affect the extraction parameters. Most phenolic compounds are extracted in high yields when using acidic NADES containing organic acids, since the extraction in aqueous solution is quantitative at $\mathrm{pH}<\mathrm{pK}_{\mathrm{a}}$ for neutral phenolic compounds.

Curcuminoids in Curcuma sp., which include curcumin (CUR), desmethoxycurcumin (DMC), and bisdemethoxycurcumin (BDMC), have numerous bioactive applications such as anti-inflammatory [14], antioxidant [14], anticarcinogenic [14-15], antibacterial [14], and antiinfection [14]. Curcuminoids can be extracted from C. longa, C. xanthorrhiza, and C. zedoaria.
In particular, the curcuminoid content in C. longa and C. zedoaria, which belongs to the Zingiberaceae or ginger family, is $3-5$ wt.\% and 6-16 wt.\%, respectively [14]. Unfortunately, to date, the use of curcuminoids as a medicinal compound is still quite limited, even though CUR has been reported to be safe for consumption up to the dose of $8 \mathrm{~g} /$ day [14]. It should be noted that the solubility of curcuminoids in water is low, i.e. $4 \mathrm{ppb}$ $(4 \mu \mathrm{g} / \mathrm{L})$ at $\mathrm{pH}=7.3[14]$ or only $3.10^{-8} \mathrm{M}$ as reported by Tonnensen [16], which limits their bioavailability. In this regard, the use of NADES as water-based environmentally friendly solvents constitutes an interesting alternative for overcoming the low solubility of CUR in water [7-8]. The application of NADES in the extraction of curcuminoids from C. zedoaria has been reported [7]. Eleven different types of NADES were evaluated, and all of them showed a high solubilizing capacity for CUR at $40^{\circ} \mathrm{C}$ within $24 \mathrm{~h}$ of extraction time. Their capacity was higher than that of common organic solvents such as methanol $(\mathrm{MeOH})$, ethanol $(\mathrm{EtOH})$, and water [7]. The curcuminoid yields were $0.269,0.163$, $0.124,0.131$, and $0.14 \mathrm{mg} / \mathrm{g}$ for fructose-glucose-water (FG- $\left.\mathrm{H}_{2} \mathrm{O}, 1: 1: 7\right)$, citric acid-sucrose-water $\left(\mathrm{CAS}-\mathrm{H}_{2} \mathrm{O}\right.$, 1:2:15 and 1:2:79), and malic acid-sucrose-water (MAS$\mathrm{H}_{2} \mathrm{O}, 1: 1: 11$ and 1:1:18), respectively. The solubility of curcuminoids in NADES was further enhanced by lowering the viscosity of NADES, which was achieved by varying the water content (up to 70 wt.\%) in $\mathrm{CAS}-\mathrm{H}_{2} \mathrm{O}$ and $\mathrm{MAS}-\mathrm{H}_{2} \mathrm{O}$ [8]. It was shown that the solubility of curcuminoids in these NADES was not affected by the water content but by the constituents of NADES $[6,8]$.

For the extraction of polyphenolics and curcuminoids from Curcuma sp., a batch stirrer extractor is commonly used $[6-8,18]$. In these systems, at the end of the extraction process, mixtures of extracted curcuminoids, NADES, and powder materials are obtained in the form of a slurry. This is also the case when using nonconventional methods of extraction such as microwave-assisted extraction, ultrasoundassisted extraction [18-19], and high pressurized liquid extraction [20-21]. Hence, separation and purification processes are required after the extraction, especially to obtain a high purity grade of the extracted compounds. 
Despite the extensive research on the application of NADES as solvents for the extraction of bioactive compounds, the development of an efficient separation method to purify the desired compounds, such as curcuminoids, from the NADES matrix after extraction is still required. Generally, the obtained extract is directly analyzed by either high-performance liquid chromatography or UV-vis spectrophotometry. Herein, two purification methods based on classical column chromatography (CCC) and solid-phase extraction (SPE) are developed for the purification of curcuminoids from the NADES matrix after extraction. Choline chloridemalic acid-water $\left(\mathrm{CCMA}-\mathrm{H}_{2} \mathrm{O}\right)$ or choline chloridecitric acid-water $\left(\mathrm{CCCA}-\mathrm{H}_{2} \mathrm{O}\right)$ in the ratio of $1: 1: 18$ were selected as NADES models due to their high solubility and stabilization capability for curcuminoids [7-8]. Moreover, NADES based on choline chloride and organic acids are reported to be less toxic and biodegradable [2].

\section{- EXPERIMENTAL SECTION}

\section{Materials}

Synthetic curcuminoids and analytical grade solvents of high purity such as glacial acetic acid (HOAc), $n$-hexane, dichloromethane (DCM), chloroform $\left(\mathrm{CHCl}_{3}\right)$, acetonitrile $(\mathrm{MeCN})$, ethanol $(\mathrm{EtOH})$, iso-propanol (IPA), and methanol (MeOH) were commercially purchased from Merck (Darmstadt, Germany). The synthetic curcuminoids were used as the standard for quantitative analysis. The individual component of NADES that consists of choline chloride (CC) and malic acid (MA), were purchased from Sigma Aldrich (St. Louis, MO, USA). Food-grade citric acid (CA) was purchased from the Surabaya local market (Gajah, Jakarta, Indonesia) while buffer solution $(\mathrm{pH}=2.0)$ was purchased from Mediss (India). Filter paper (no. 589/2) with a pore size of 4-12 $\mu \mathrm{m}$ was purchased from Whatman (Germany). For the column chromatography, silica gel $60 \AA$ (52-73 $)$, 70-230 mesh, with a particle size of 63-200 $\mu \mathrm{m}$ was purchased from Merck (Darmstadt, Germany). A silica gel plate $60 \mathrm{~F} 254$ with a size of $20 \times 20 \mathrm{~cm}$ (Merck, Darmstadt, Germany) was used for qualitative analysis by thin layer chromatography (TLC), and the cartridge for
SPE, Hypersep $\mathrm{C}_{18} 500 \mathrm{mg} / 2.8 \mathrm{~mL}$, was purchased from Thermo Fisher Scientific (USA).

\section{Instrumentation}

The instruments used were spectrophotometer UV-vis T-60 (PG Instrument, Leicestershire, UK), analytical balance (OHAUSS), and vacuum pump (Chem-Tech, USA).

\section{Procedure}

\section{Sample preparation}

All the NADES used in this study were prepared according to a previously reported method [8]. To mimic a CUR extract, an artificial sample was prepared by dissolving curcuminoids in NADES with a solvent per feed ratio $(\mathrm{S} / \mathrm{F})$ of $5 / 10 \mathrm{~mL}$ NADES/mg curcuminoids. As much as $10 \pm 0.1 \mathrm{mg}$ of curcuminoids was used for the CCC purification method, whereas $3 \pm$ $0.1 \mathrm{mg}$ curcuminoids was used for the SPE method because the maximum loading capacity of the SPE cartridge is $2.8 \mathrm{~mL}$. The mixture was magnetically stirred at $40{ }^{\circ} \mathrm{C}$ until all the curcuminoids were well dissolved. The standard sample of curcuminoids was used in both the CCMA- $\mathrm{H}_{2} \mathrm{O}$ and CCCA- $\mathrm{H}_{2} \mathrm{O}$ at a molar ratio of 1:1:18.

\section{Quantitative analysis of curcuminoids by UV-vis spectrophotometry}

The curcuminoids were quantitatively analyzed using UV-vis spectrophotometer. Briefly, $100 \mu \mathrm{L}$ of sample was accurately diluted into $\mathrm{MeOH} / 3 \% \mathrm{HOAc}$ to obtain a volume of $2 \mathrm{~mL}$. The $\mathrm{pH}$ was adjusted at 1.8-2.0 using a $\mathrm{pH}=2$ buffer solution. Then, the sample was subjected to $\mathrm{UV}$-vis analysis at $420 \mathrm{~nm}\left(\lambda_{\max }\right)$. The obtained values of absorbance were then converted to curcuminoids concentration via a previously prepared calibration curve. The recovery of curcuminoids was calculated as the amount of obtained curcuminoids divided by the initial weight of the powder of C. zedoaria.

Prior to the quantitative calculation of curcuminoids, a calibration curve of curcuminoids was prepared. Seven solutions of curcuminoids with concentrations of $1,2,3,4,5,6$, and $7 \mathrm{ppm}$ were prepared in serial dilutions [22]. A mixture of $\mathrm{MeOH} / \mathrm{HOAc}$ 
(99:1, v/v) was used as a dilution solvent. Each sample was kept at $\mathrm{pH}=1.8-2.0$ using the buffer solution. As much as $1 \mathrm{mg}$ of curcuminoid standard was precisely weighed and dissolved into $10 \mathrm{~mL}$ NADES of CCMA- $\mathrm{H}_{2} \mathrm{O}(1: 1: 18)$ or $\mathrm{CCCA}-\mathrm{H}_{2} \mathrm{O} \quad(1: 1: 18)$, in a volumetric flask. The concentration was set at $100 \mathrm{ppm}(100 \mathrm{mg} / \mathrm{L})$, and the absorbance of each sample was subsequently measured in triplicates. The limit of detection (LOD) and the limit of quantification (LOQ) were also calculated for each calibration curve [22].

\section{Curcuminoids purification using the classical column chromatography method}

The silica gel used as the stationary phase was poured into a glass column of $2 \mathrm{~cm}$ in diameter. At first, the column was conditioned by eluting $\mathrm{MeOH}$ through the silica bed, followed by closing the top of the column using cotton wetted with $\mathrm{MeOH}$ and allowing the system to stand for hours. The polarity of the silica was increased in the presence of $\mathrm{MeOH}$. Thus, the polar constituents of NADES (CC, CA, and MA) were easily separated from the sample.

An artificial curcuminoids sample containing $10 \mathrm{mg}$ of curcuminoids in $5 \mathrm{~mL}$ NADES $(\mathrm{S} / \mathrm{F}$ ratio $=5 / 10)$ was then loaded into the column. The NADES constituents were retained in the silica. Initially, $25 \mathrm{~mL}$ of $\mathrm{MeCN} / 0.1 \%$ HOAc ( $/ / v)$ was eluted four times, obtaining four fractions for each solvent mixture. By adding HOAc in the mobile phase, an acidic eluent was generated. Afterwards, $\mathrm{H}_{2} \mathrm{O} / 0.1 \%$ HOAc (v/v), and IPA/0.1\% HOAc (v/v) were sequentially eluted. Each mobile phase mixture was eluted four times, producing a total volume of $100 \mathrm{~mL}$. The process was repeated twice for each NADES, namely, CCMA- $\mathrm{H}_{2} \mathrm{O}(1: 1: 18)$ and CCCA- $\mathrm{H}_{2} \mathrm{O}$ (1:1:18).

\section{Curcuminoids purification using the solid-phase extraction method}

The SPE method was selected to enhance the recovery of curcuminoids. A less polar cartridge type $\left(\mathrm{C}_{18}\right)$ was used to separate the NADES matrix from the sample. The hydrophilic tail of the silica backbone in the SPE cartridge was modified using $\mathrm{C}_{8}-\mathrm{C}_{18}$ cartridges. The SPE cartridge polarity was relatively lower than that of the silica gel in the CCC method. A reverse approach of separation was applied in the SPE method compared with the CCC method.

The purification of curcuminoids by SPE comprised of four steps which include conditioning, loading, washing, and eluting, using different mobile phase mixtures in each step. For the elution process, 1.5 $\mathrm{mL}$ of mobile phase was used. A mixture of $\mathrm{MeOH} / 0.05 \% \mathrm{HOAc}(\mathrm{v} / \mathrm{v})$ and $\mathrm{H}_{2} \mathrm{O} / 0.05 \% \mathrm{HOAc}(\mathrm{v} / \mathrm{v})$ was subsequently eluted using an SPE Hypersep $\mathrm{C}_{18}$ cartridge during the conditioning step. At the loading stage, $1.5 \mathrm{~mL}$ of the sample containing NADES and curcuminoids (solid/fluids ratio of $5 \mathrm{~g} / 10 \mathrm{~mL}$ ) was poured into the cartridge. Then, a mixture of $\mathrm{H}_{2} \mathrm{O} / 0.05 \%$ HOAc (v/v) was used during the washing stage, and 1 $\mathrm{mL}$ of an $\mathrm{MeOH} / \mathrm{MeCN}(1: 1, \mathrm{v} / \mathrm{v})$ solution was subsequently eluted to extract the remaining curcuminoids in the cartridge. In the eluting step, $3 \mathrm{~mL}$ of the $\mathrm{MeOH} / \mathrm{MeCN}(1: 1, \mathrm{v} / \mathrm{v})$ solution was eluted. The obtained purified extract from the successive solvent extraction was evaporated using $\mathrm{N}_{2}$. The dried curcuminoids residue was precisely weighed, and the recoveries were calculated. This process was performed in triplicates for each NADES.

\section{Qualitative analysis of curcuminoids by TLC}

To monitor the progress of curcuminoids purification by CCC, the TLC analysis was performed. The qualitative analysis was conducted using DCM/MeOH $(95: 5, \mathrm{v} / \mathrm{v})$ as the mobile phase [23]. The spots were observed under UV light at $365 \mathrm{~nm}$. The visualized spots were marked, and the retardation factor (Rf) was calculated.

\section{- RESULTS AND DISCUSSION}

Prior to conducting purification processes of curcuminoids from NADES matrices; for quantitative purposes, the following calibration curves were produced: $y=0.125 x-0.058\left(R^{2}=0.99\right)$ and $y=0.108 x-0.056\left(R^{2}\right.$ $=0.99)$ for CCMA $-\mathrm{H}_{2} \mathrm{O}(1: 1: 18)$ and CCCA $-\mathrm{H}_{2} \mathrm{O}$ (1:1:18), respectively (Fig. 1). The LOD and LOQ were also calculated, and the results are shown in Table 1.

In CUR, the keto-enol tautomeric equilibrium occurs due to the presence of a hydroxyl $(-\mathrm{OH})$ groups in 
Table 1. Limit of detection (LOD) and limit of quantification (LOQ) for the NADES CCMA- $\mathrm{H}_{2} \mathrm{O}(1: 1: 18)$ and CCCA$\mathrm{H}_{2} \mathrm{O}(1: 1: 18)$

\begin{tabular}{ccc}
\hline Type of NADES & $\begin{array}{c}\text { Limit of detection } \\
(\text { LOD }, \text { ppm })\end{array}$ & $\begin{array}{c}\text { Limit of quantification } \\
(\text { LOQ, ppm) }\end{array}$ \\
\hline CCMA- $\mathrm{H}_{2} \mathrm{O}(1: 1: 18)$ & 0.862 & 2.613 \\
CCCA- $\mathrm{H}_{2} \mathrm{O}(1: 1: 18)$ & 2.719 & 8.239 \\
\hline
\end{tabular}
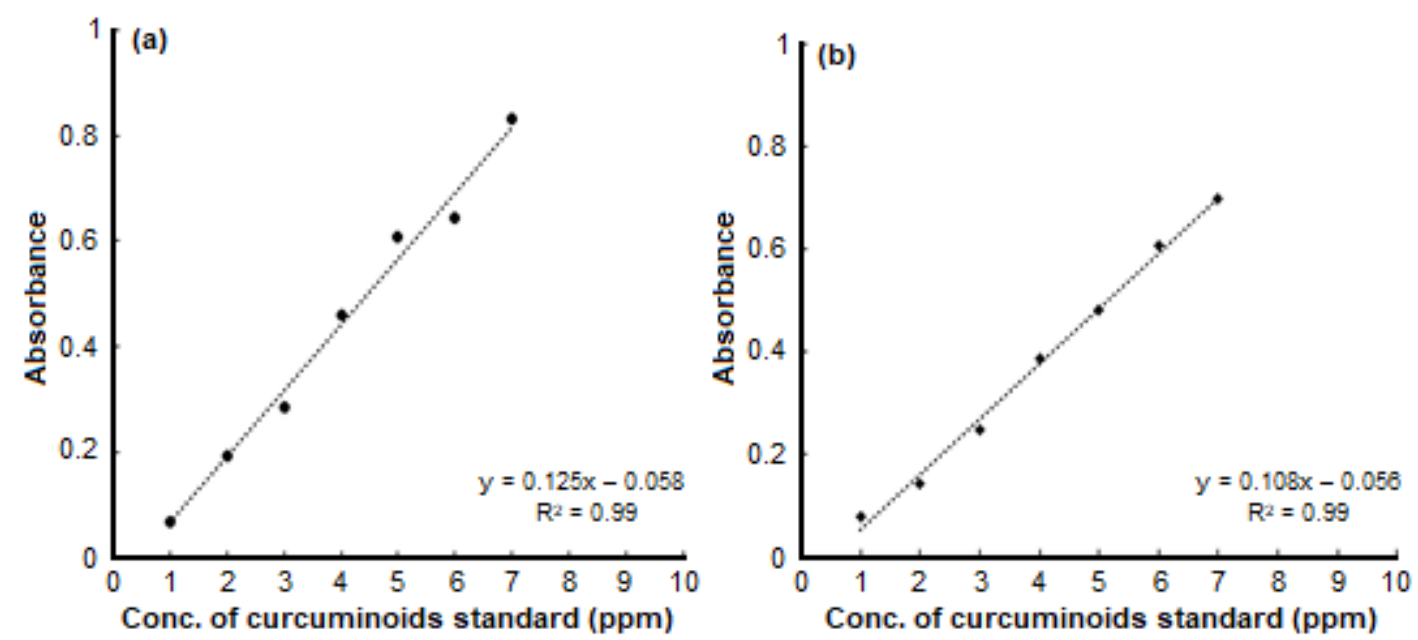

Fig 1. Calibration curves of curcuminoids in NADES matrices: (a) CCMA- $\mathrm{H}_{2} \mathrm{O}(1: 1: 18)$ and (b) $\mathrm{CCCA}-\mathrm{H}_{2} \mathrm{O}(1: 1: 18)$

the alkene moiety $(\mathrm{C}=\mathrm{C})$ of CUR. The keto form of CUR is more frequently found in the solid phase than the liquid phase, whereas the enol form is predominantly observed in the liquid phase or in solution [24]. The keto form is thermodynamically more stable than the enol form by $48 \mathrm{~kJ} / \mathrm{mol}$ due to the presence of $\mathrm{C}-\mathrm{H}, \mathrm{C}-\mathrm{C}$, and $\mathrm{C}=\mathrm{O}$ bonds [25], whereas the enol structure has $\mathrm{C}=\mathrm{C}, \mathrm{C}-\mathrm{O}$, and $\mathrm{O}-\mathrm{H}$ bonds [25]. However, the enol tautomer can be stabilized by hydrogen bonds. Moreover, the keto form of CUR is barely influenced by changes in the $\mathrm{pH}$ or the temperature. Thus, CUR in the solid phase is more stable and can be stored for a longer time than in the liquid phase. Consequently, it is more convenient to store the extracted curcuminoids from the NADES matrix in the solid phase.

Recrystallization has been previously attempted to extract curcuminoids directly from the NADES matrix (CCMA- $\mathrm{H}_{2} \mathrm{O}=1: 1: 18$ or CCCA $-\mathrm{H}_{2} \mathrm{O}=1: 1: 18$ ), by using different volumes or molar ratios of iso-propanol $/ n$ hexane $(1: 1,1: 1.5,1: 2,2: 1$, or 2.5:1) as recrystallization solvents [26]. Iso-propanol (IPA) was used due to its solubilization capability for curcuminoids, and $n$-hexane was used as an antisolvent to induce precipitation [2627]. However, due to the presence of NADES constituents (CC, CA, and MA), a homogenous mixture of curcuminoids, NADES, and IPA was observed, and attempts to perform the recrystallization failed [26]. Since CC-1,2-propanediol was reported to form a NADES [5], a CC-CA-IPA- $\mathrm{H}_{2} \mathrm{O}$ mixture could be expected to form a new NADES mixture. Yadav [29] successfully recrystallized CUR using an artificial sample of a CUR crystalline standard dissolved in $\mathrm{EtOH}$ from IPA- $n$-hexane $(1: 5, \mathrm{v} / \mathrm{v})$ at $30^{\circ} \mathrm{C}$, obtaining amorphous CUR. Consequently, NADES constituents should be removed first before the crystallization step of curcuminoids to eliminate the compounds that interfere with the crystallization process. To achieve this, we used the CCC and SPE methods, in which the flexibility in selecting both stationary and mobile phases of eluent mixtures enables an easy tuning of the polarity of the purification method for different types of NADES.

\section{Purification Using the CCC Method}

We started our investigation by evaluating the CCC method for the separation of curcuminoids from 
the NADES constituents, namely, CC, CA, and MA. The stationary phase was highly polar due to the presence of oxygen atoms in the silica gel. By adding HOAc to the mobile phase of CCC, an acidic eluent was generated. Curcuminoids are in the keto form at $\mathrm{pH}=3-7[16,24]$ and have relatively low polarity [23]. In acidic condition, the polarity of curcuminoids is low, and their solubility in $\mathrm{MeCN}$ is greater. Accordingly, curcuminoids were quantitively detected to be present in the first four extracts, i.e. $1^{\text {st }}-4^{\text {th }}$ fractions (Table 2), indicating that curcuminoids were successfully eluted with $\mathrm{MeCN} / 0.1 \%$ $\mathrm{HOAc}(\mathrm{v} / \mathrm{v})$ as the first mobile phase in acidic condition.

When the second mobile phase of $\mathrm{H}_{2} \mathrm{O} / 0.1 \%$ HOAc $(\mathrm{v} / \mathrm{v})$ was eluted, some curcuminoids were observed in the $5^{\text {th }}-8^{\text {th }}$ fractions for CCCA- $\mathrm{H}_{2} \mathrm{O}$ (1:1:18). However, for CCMA- $\mathrm{H}_{2} \mathrm{O}$ (1:1:18), no curcuminoids were observed in the $8^{\text {th }}$ fraction, and relatively low curcuminoid content was found in the $7^{\text {th }}$ fraction (Table 2). This is presumably because $\mathrm{CA}$ has three carbonyl groups $\left(\mathrm{pKa}_{1}\right.$ $=3.13, \mathrm{pKa}_{2}=4.76$, and $\mathrm{pKa}_{3}=6.39$ ), whereas MA only has two carbonyl groups $\left(\mathrm{pKa}_{1}=3.40\right.$ and $\left.\mathrm{pKa}_{2}=5.20\right)$; consequently, curcuminoids would form more hydrogen bonds with CCCA- $\mathrm{H}_{2} \mathrm{O}$ than with CCMA$\mathrm{H}_{2} \mathrm{O}$. Therefore, more volume of the mobile phase was required to disrupt the hydrogen bonds and to remove the curcuminoids from the NADES matrix.

When IPA/0.1\% HOAc (v/v) was eluted, no curcuminoids were obtained. All the curcuminoids were, presumably, already eluted out, or the content of purified curcuminoids was below both the LOD and the LOQ. From the CCCA- $\mathrm{H}_{2} \mathrm{O}$ and $\mathrm{CCMA}-\mathrm{H}_{2} \mathrm{O}$ matrices, $5.19 \pm 0.054 \mathrm{mg}$ and $6.10 \pm 0.242 \mathrm{mg}$ of curcuminoids

Table 2. Amount of curcuminoids in each fraction of column chromatography purification method

\begin{tabular}{|c|c|c|c|c|c|}
\hline \multirow{3}{*}{ Type of NADES } & \multirow{3}{*}{ Fraction } & \multicolumn{3}{|c|}{ Retardation Factor $\left(\mathrm{R}_{\mathrm{f}}\right)^{*}$} & \multirow{3}{*}{$\begin{array}{l}\text { Curcuminoids } \\
\qquad(\mathrm{mg})\end{array}$} \\
\hline & & $\mathrm{C}$ & DMC & BDMC & \\
\hline & & 0.89 & 0.71 & 0.53 & \\
\hline \multirow{11}{*}{$\begin{array}{c}\text { CCCA- } \mathrm{H}_{2} \mathrm{O} \\
(1: 1: 18)\end{array}$} & $1^{*}$ & - & - & - & - \\
\hline & $2^{*}$ & + & ++ & ++ & $1.21 \pm 0.004$ \\
\hline & $3^{*}$ & ++ & ++ & + & $1.67 \pm 0.021$ \\
\hline & $4^{\star}$ & ++ & + & + & $1.00 \pm 0.013$ \\
\hline & $5^{* *}$ & + & + & - & $0.17 \pm 0.003$ \\
\hline & $6^{* *}$ & + & + & - & $0.97 \pm 0.011$ \\
\hline & $7^{* *}$ & + & - & - & $0.11 \pm 0.001$ \\
\hline & $8^{\star *}$ & + & - & - & $0.06 \pm 0.001$ \\
\hline & $9-12^{\star * *}$ & - & - & - & - \\
\hline & \multirow{2}{*}{\multicolumn{4}{|c|}{$\begin{array}{r}\text { Total Curcuminoids } \\
\text { (recovery) }\end{array}$}} & $5.19 \pm 0.054$ \\
\hline & & & & & $(51.9 \%)$ \\
\hline \multirow{11}{*}{$\begin{array}{c}\text { CCMA- } \mathrm{H}_{2} \mathrm{O} \\
(1: 1: 18)\end{array}$} & $1^{*}$ & - & - & - & - \\
\hline & $2^{*}$ & + & ++ & ++ & $1.40 \pm 0.045$ \\
\hline & $3^{*}$ & ++ & ++ & ++ & $1.27 \pm 0.031$ \\
\hline & $4^{*}$ & ++ & ++ & + & $1.05 \pm 0.088$ \\
\hline & $5^{\star *}$ & ++ & ++ & + & $1.38 \pm 0.020$ \\
\hline & $6^{* *}$ & + & + & - & $0.95 \pm 0.057$ \\
\hline & $7^{\star *}$ & + & - & - & $0.05 \pm 0.001$ \\
\hline & $8^{* *}$ & - & - & - & - \\
\hline & $9-12^{* * *}$ & - & - & - & - \\
\hline & \multirow{2}{*}{\multicolumn{4}{|c|}{$\begin{array}{r}\text { Total Curcuminoids } \\
\text { (recovery) }\end{array}$}} & $6.10 \pm 0.242$ \\
\hline & & & & & $(61.0 \%)$ \\
\hline
\end{tabular}

Orchidea Rachmaniah et al. 
were recovered, respectively, indicating that $51.9 \%$ and $61.0 \%$ of curcuminoids were recovered by the CCC purification method.

The obtained recovery of curcuminoids was higher than that reported by Kulkarni [28], in which a CCC with silica gel as a bed resin was used to purify an extract of crude curcuminoids from turmeric, C. longa $\mathrm{L}$. A mixture of $\mathrm{CHCl}_{3} / \mathrm{MeOH}$ was used as an eluent, starting with a 95:5 ratio and increasing the $\mathrm{MeOH}$ content to increase the polarity. The recovery of $5.6 \%$ (from $0.34 \mathrm{~g}$ of purified extract) was achieved.

The CCC method was previously used by Revathy to purify a crude extract of curcuminoids that was obtained after precipitating with petroleum ether in an acetone extract [29]. Silica gel was also used as a bed resin, producing 95 fractions of $40 \mathrm{~mL}$ each. The recovery of CUR was $84 \%$, which was mostly purified at the initial fractions (ca. 1-31). The $\mathrm{CHCl}_{3} / \mathrm{MeOH}$ mixture with different volume compositions was applied to enhance the polarity. The obtained recovery of CUR was higher because the initial extract was relatively pure and soluble in acetone, a less polar solvent. The CUR is less polar than BDMC and DMC (the two other curcuminoids in Curcuma) [16,23], with BDMC being the most polar $[23,29]$. Therefore, a high proportion of $\mathrm{CHCl}_{3}$ was used at the initial stage of elution, i.e., $\mathrm{CHCl}_{3} / \mathrm{MeOH}$ (19:1, $\mathrm{v} / \mathrm{v})$. In the work of Revathy [29], no polar compounds accompanied the crude extract of curcuminoids as impurities. Hence, a different separation approach based on CCC was applied, reaching higher recovery of CUR.

Heffernan [30] also applied the CCC method for the purification of curcuminoids from the remaining mother liquor of crystallization. The mixture of $\mathrm{CHCl}_{3} / \mathrm{MeOH}$ was also used as a mobile phase. The pure CUR was successfully obtained when chloroform was eluted into the CCC. Upon increasing the methanol ratio in the eluent, DMC was identified in the purified extract. Finally, BDMC was obtained when the $\mathrm{CHCl}_{3} / \mathrm{MeOH}$ mixture with the ratio of 7:8 $(\mathrm{v} / \mathrm{v})$ was applied [28], which further confirms the higher polarity of BDMC compared with CUR and DMC. In the reports by Revathy [29] and Heffernan [30], CUR was eluted first using a less polar solvent, and the polarity of the mobile phase was subsequently increased to elute DMC and BDMC consecutively. As in the case of Revathy [29] and Heffernan [30], in the present study, curcuminoids were first eluted using $\mathrm{MeCN} / 0.1 \%$ HOAc. By increasing the polarity of the mobile phase using water and isopropanol instead of $\mathrm{MeCN}$, impurities such as the NADES constituents that were retained in the column were then washed out.

\section{Purification Using the SPE Method}

Curcuminoids are expected to be retained on the $\mathrm{C}_{18}$ SPE cartridge because of their low polarity. In the contrary, water and the NADES constituents (CC, CA, and MA) can be expected to elute first. Therefore, the mobile phase was first used to wash out all the NADES constituents from the cartridge. Subsequently, another eluent was used to elute the target curcuminoids, which were then separated from NADES.

Initially, the cartridge was conditioned using $1.5 \mathrm{~mL}$ $\mathrm{MeOH} / 0.05 \% \mathrm{HOAc}(\mathrm{v} / \mathrm{v})$ and $1.5 \mathrm{~mL} \mathrm{H}_{2} \mathrm{O} / 0.05 \%$ HOAc (v/v) (Fig. 2(C)). Thus, the resin was in acidic condition. After $1.5 \mathrm{~mL}$ of sample containing $2 \mathrm{mg} / \mathrm{mL}$ of curcuminoids $(\mathrm{S} / \mathrm{F}=5 / 10, \mathrm{mg} / \mathrm{mL})$ was loaded, curcuminoids were retained in the cartridge (Fig. 2(L)) and remained separated from the NADES impurities owing to the acidic condition. Subsequently, the washing stage was started by eluting $\mathrm{H}_{2} \mathrm{O} / 0.05 \% \mathrm{HOAc}(\mathrm{v} / \mathrm{v})$. As can be seen in Fig. 2(W), a partial elution of curcuminoids through the cartridge occurred (but they were not washed out) since the acidic condition of the SPE cartridge resin caused the curcuminoids to strongly bind with the cartridge. Therefore, the NADES constituents and water were eluted, whereas the less polar compounds, the curcuminoids, were retained. The hydrogen bonds between NADES and the curcuminoids were cleaved due to the dilution effect of water in the eluent.

The eluting step was then conducted by passing $\mathrm{MeOH} / \mathrm{MeCN}(1: 1, \mathrm{v} / \mathrm{v})$. First, $1 \mathrm{~mL}$ of eluent was used (Fig. $2\left(1^{\text {st }} \mathrm{E}\right)$ ), and the curcuminoids eluted from the SPE cartridge were collected in a vial. Then, the second and the third eluent were passed, making a total of $3 \mathrm{~mL}$. All the curcuminoids were recovered when the SPE cartridge turned white (Fig. $2\left(3^{\text {rd }} \mathrm{E}\right)$ ). The results of this 


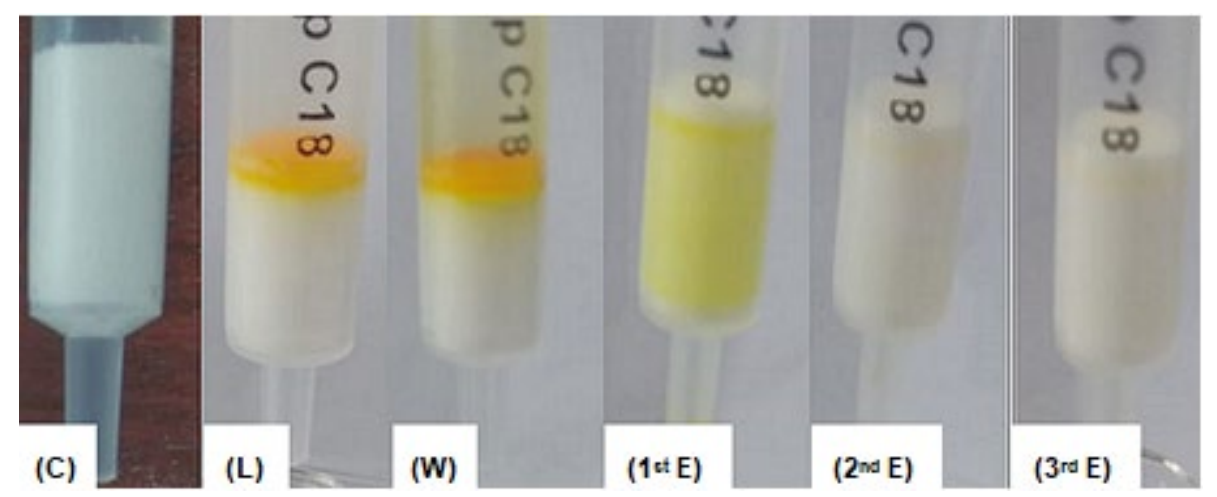

Fig 2. Images of the cartridges used in the solid phase extraction method: conditioning (C), loading (L), washing (W), and eluting (E) steps

procedure are summarized in Table 3. The first elution at the eluting step gave the highest recovery of curcuminoids. Then, the remaining curcuminoids retained in the cartridge were eluted in the other two elution steps. All the curcuminoids were thereby recovered. As can be seen from Table 3, only a small amount of curcuminoids was recovered in the third elution. The TLC chromatogram of the recovery of curcuminoids from the NADES matrices by SPE is shown in Fig. 3.

The purified curcuminoid solutions that were collected in vials were subsequently dried by passing $\mathrm{N}_{2}$ gas. Using the present SPE method, $2.258 \pm 0.017$ and $2.202 \pm 0.021 \mathrm{mg}$ of curcuminoids were successfully recovered from CCCA- $\mathrm{H}_{2} \mathrm{O}$ and CCMA- $\mathrm{H}_{2} \mathrm{O}$, respectively (Table 3). Hence, $75.27 \%$ and $73.40 \%$ recoveries of curcuminoids were obtained, which were

Table 3. The purified curcuminoids from NADES matrices using solid phase extraction (SPE)

\begin{tabular}{lcr}
\hline NADES Type & Eluent & Curcuminoids $(\mathrm{mg})^{\lambda}$ \\
\hline & $1^{\text {st }}$ & $2.190 \pm 0.01700$ \\
CCCA-H ${ }_{2} \mathrm{O}(1: 1: 18)$ & $2^{\text {nd }}$ & $0.060 \pm 0.00040$ \\
& $3^{\text {rd }}$ & $0.004 \pm 0.00002$ \\
\hline & Total (mg) & $2.258 \pm 0.01700$ \\
& (recovery) & $75.27 \%$ \\
\hline & $1^{\text {st }}$ & $2.140 \pm 0.0090$ \\
CCMA- $_{2} \mathrm{O}(1: 1: 18)$ & $2^{\text {nd }}$ & $0.060 \pm 0.0030$ \\
& $3^{\text {rd }}$ & $0.005 \pm 0.0027$ \\
\hline & Total (mg) & $2.202 \pm 0.0210$ \\
& (recovery) & $73.40 \%$ \\
\hline
\end{tabular}

${ }^{\lambda}$ Each purification was conducted in triplicate
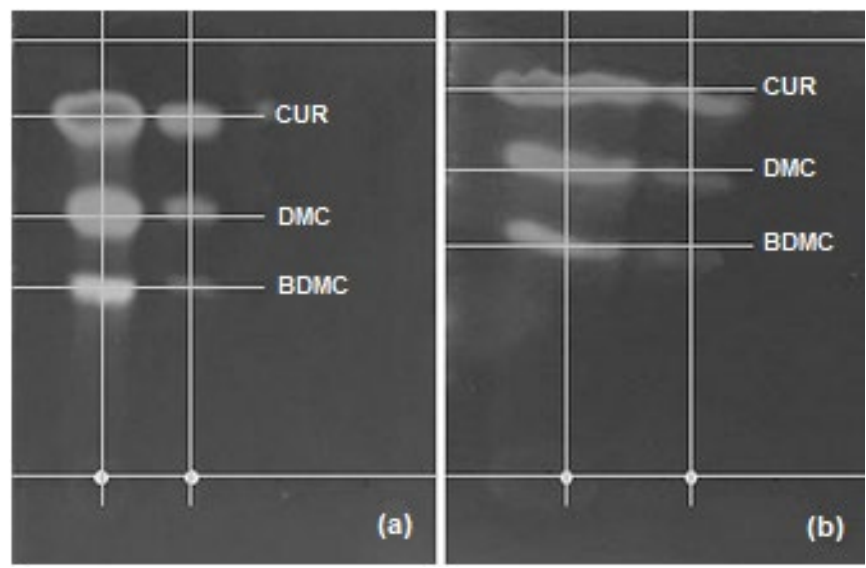

Fig 3. The TLC chromatogram of the recovered curcuminoids from NADES matrices by solid phase extraction: (a) CCCA- $\mathrm{H}_{2} \mathrm{O}$ (1:1:18) and (b) CCMA$\mathrm{H}_{2} \mathrm{O}(1: 1: 18)$

relatively higher than those of the CCC method. Pushpakumari et al. [31] used Diaion HP 20 resin to purify an enriched sample of curcuminoids from spent turmeric oleoresin by chromatography [31]. When 80 wt.\% of $\mathrm{MeOH}$ was used as the eluent, CUR, DMC, and BDMC were successfully obtained in the purity of $98.4 \%$, $97.1 \%$, and $97.3 \%$, respectively. Despite the high recovery and purity of curcuminoids reported by Revathy [29], Haffernan [30], and Pushpakumari [31], it should be noted that relatively pure extracts of curcuminoids were used in these experiments. Therefore, it should be mentioned that curcuminoids were able to be separated from NADES matrices for the first time in the present work. 


\section{- CONCLUSION}

The purification of the curcuminoids from NADES matrices of CCCA- $\mathrm{H}_{2} \mathrm{O} \quad(1: 1: 18)$ and CCMA- $\mathrm{H}_{2} \mathrm{O}$ (1:1:18) was successfully conducted by CCC and SPE methods. The addition of glacial acetic acid in the mobile phase afforded a slightly acidic condition, which was conducive to enhance the curcuminoids recovery. The CCC method only yielded a recovery of curcuminoids of $51.9 \%$ and $61.0 \%$ for CCCA- $\mathrm{H}_{2} \mathrm{O}$ and CCMA- $\mathrm{H}_{2} \mathrm{O}$, respectively, and higher recoveries of $75.27 \%$ and $73.40 \%$ were obtained with the SPE method. Both methods were effectively applied in laboratory scale. For large-scale application, other methods would be required.

\section{- REFERENCES}

[1] Choi, Y.H., van Spronsen, J., Dai, Y., Verberne, M., Hollmann, F., Arends, I.W.C.E., Witkamp, G.J., and Verpoorte, R., 2011, Are natural deep eutectic solvents the missing link in understanding cellular metabolism and physiology?, Plant Physiol., 156 (4), 1701-1705.

[2] Paiva, A., Craveiro, R., Aroso, I., Martins, M., Reis, R.L., and Duarte, A.R.C., 2014, Natural deep eutectic solvents - Solvents for the $21^{\text {st }}$ century, ACS Sustainable Chem. Eng., 2 (5), 1063-1071.

[3] Gorke, J.T., Srienc, F., and Kazlauskas, R.J., 2008, Hydrolase-catalyzed biotransformations in deep eutectic solvents, Chem. Commun., 10, 1235-1237.

[4] Dai, Y., van Spronsen, J., Witkamp, G.J., Verpoorte, R., and Choi, Y.H., 2013, Natural deep eutectic solvents as new potential media for green technology, Anal. Chim. Acta, 766, 61-68.

[5] Dai, Y., Witkamp, G.J., Verpoorte, R., and Choi, Y.H., 2013, Natural deep eutectic solvents as new potential media for phenolic metabolites in Carthamus tinctorius L., Anal. Chem., 85 (13), 6272-6278.

[6] Liu, Y., Li, J., Fu, R., Zhang, L., Wang, D., and Wang, S., 2019, Enhanced extraction of natural pigments from Curcuma longa L. using natural deep eutectic solvents, Ind. Crops Prod., 140, 111620.

[7] Zullaikah, S., Rachmaniah, O., Utomo A.T., Niawati, H., and Ju, Y.H., 2018, "Green Separation of Bioactive Natural Products using Liquefied Mixture of Solids" in Green Chemistry, Eds. Saleh, H.E.D.M. and Koller, M., InTechOpen, London, UK.

[8] Rachmaniah, O., Fazriyah, L.J., Seftiyani, N.H., and Rachimoellah, M., 2018, Tailoring properties of acidic types of Natural Deep Eutectics Solvents (NADES): Enhanced solubility of curcuminoids from Curcuma zedoaria, MATEC Web Conf., 156, 01011.

[9] Chanioti, S., and Tzia, C., 2018, Extraction of phenolic compounds from olive pomace by using natural deep eutectic solvents and innovative extraction techniques, Innovative Food Sci. Emerging Technol., 48, 228-239.

[10] Panić, M., Radić Stojković, M., Kraljić, K., Škevin, D., Radojčić Redovniković, I., Gaurina Srček, V., and Radošević, K., 2019, Ready-to-use green polyphenolic extracts from food by-products, Food Chem., 283, 628-636.

[11] Hamany, D.C.Y., Piater, L.A., Steenkamp, P.A., Madala, N.E., and Dubery, I.A., 2018, Differential extraction of phytochemicals from the multipurpose tree, Moringa oleifera, using green extraction solvents, S. Afr. J. Bot., 115, 81-89.

[12] Ivanović, M., Alañón, M.E., Arráez-Román, D., and Segura-Carretero, A., 2018, Enhanced and green extraction of bioactive compounds from Lippia citriodora by tailor-made natural deep eutectic solvents, Food Res. Int., 111, 67-76.

[13] Miličević, N., Panić, M., Valinger, D., Cvjetko Bubalo, M., Benković, M., Jurina, T., Gajdoš Kljusurić, J., Radojčić Redovniković, I., and Jurinjak Tušek, A., 2020, Development of continuously operated aqueous two-phase microextraction process using natural deep eutectic solvents, Sep. Purif. Technol., 244, 116746.

[14] Kocaadam, B., and Şanlier, N., 2017, Curcumin, an active component of turmeric (Curcuma longa), and its effects on health, Crit. Rev. Food Sci. Nutr., 57 (13), 2889-2895.

[15] Patra, D., and Sleem, F., 2013, A new method for pH triggered curcumin release by applying poly(Llysine) mediated nanoparticle-congregation, Anal. Chim. Acta, 795, 60-68. 
[16] Tønnesen, H.H., and Karlsen, J., 1985, Studies on curcumin and curcuminoids, Z. Lebensm. Unters. Forsch., 180 (5), 402-404.

[17] Patil, S.S., Bhasarkar, S., and Rathod, V.K., 2019, Extraction of curcuminoids from Curcuma longa: Comparative study between batch extraction and novel three phase partitioning, Prep. Biochem. Biotechnol., 49 (4), 407-418.

[18] Patil, S.S., Pathak, A., and Rathod, V.K., 2021, Optimization and kinetic study of ultrasound assisted deep eutectic solvent based extraction: A greener route for extraction of curcuminoids from Curcuma longa, Ultrason. Sonochem., 70, 105267.

[19] Neves, M.I.L., Strieder, M.M., Vardanega, R., Silva, E.K., and Meireles, M.A.A., 2020, Biorefinery of turmeric (Curcuma longa L.) using non-thermal and clean emerging technologies: An update on the curcumin recovery step, $R S C A d v ., 10$ (1), 112-121.

[20] Kiamahalleh, M.V., Najafpour-Darzi, G., Rahimnejad, M., Moghadamnia, A.A., and Kiamahalleh, M.V., 2016, High performance curcumin subcritical water extraction from turmeric (Curcuma longa L.), J. Chromatogr. B, 1022, 191-198.

[21] Osorio-Tobón, J.F., Carvalho, P.I.N., Rostagno, M.A., and Meireles, M.A.A., 2016, Process integration for turmeric products extraction using supercritical fluids and pressurized liquids: Economic evaluation, Food Bioprod. Process., 98, 227-235.

[22] Yuwono, M., and Indrayanto, G., 2005, Validation of chromatographic methods of analysis. In profiles of drug substances, Profiles Drug Subst., Excipients, Relat. Method., 32, 243-259.

[23] Fagundes, T.S.F., Dutra, K.D.B., Ribeiro, C.M.R., Epifanio, R.A., and Valverde, A.L., 2015, Using a Sequence of experiments with turmeric pigments from food to teach extraction, distillation, and thin layer chromatography to introductory organic chemistry students, J. Chem. Educ., 93 (2), 326-329.

[24] Nugroho, A.E., Yuniarti, N., Istyastono, E.P.,
Supardjan, S., and Hakim, L., 2006, Determination of antioxidant activity of dehydro-zingerone through hydroxyl radical scavengers using deoxyribose method, Indones. J. Pharm., 17 (3), 116-122.

[25] Istyastono, E.P., Martono, S., Pranowo, H.D., and Tahir, I., 2003, Quantitative structure activity relationship analysis of curcumin and its derivate as GST inhibitors bases on computational chemistry calculation, Indones. J. Chem., 3 (3), 179-186.

[26] Rachmaniah, O., Muhsin, M.R., Widya, A.P., and Rachimoellah, M., 2020, Process development for the enrichment of curcuminoids in the extract of ionic type of NADES, IOP Conf. Ser.: Mater. Sci. Eng., 732, 012005.

[27] Yadav, D., and Kumar, N., 2014, Nanonization of curcumin by antisolvent precipitation: Process development, characterization, freeze drying and stability performance, Int. J. Pharm., 477 (1-2), 564-577.

[28] Kulkarni, S.J., Maske, K.N., Budre, M.P., and Mahajan, R.P., 2017, Extraction and purification of curcuminoids from turmeric (Curcuma longa L.), Int. J. Pharmacol. Pharm. Technol., 1 (2), 81-84.

[29] Revathy, S., Elumalai, S., Benny, M., and Antony, B., 2011, Isolation, purification and identification of curcuminoids from turmeric (Curcuma longa L.) by column chromatography, J. Exp. Sci., 2 (7), 21-25.

[30] Heffernan, C., Ukrainczyk, M., Gamidi, R.K., Hodnett, B.K., and Rasmuson, Å.C., 2017, Extraction and purification of curcuminoids from crude curcumin by a combination of crystallization and chromatography, Org. Process Res. Dev., 21 (6), 821-826.

[31] Pushpakumari, K.N., Varghese, N., and Kottol, K., 2014, Purification and separation of individual curcuminoids from spent turmeric oleoresin, a byproduct from curcumin production industry, Int. J. Pharm. Sci. Res., 5 (8), 3246-3254. 